\title{
A Study on the Current State of the Chinese Proficiency Test (HSK) at the confucius Institute in Sofia, Bulgaria
}

Liu Ming

Beijing Foreign Studies University, China

Abstract: The Chinese Proficiency Test (HSK) is a standardized test on Chinese language skills which has been widely introduced both in and outside China and has been playing an important role in the spread of Chinese learning all over the world. In 2003 Bulgaria hosted a beginner level HSK exam, which made it the first location to hold a Chinese language proficiency test on the Balkan Peninsula and ever since it has been very successful. The current paper provides a research on the current state of the Chinese Proficiency Test (HSK) at the Confucius Institute in Sofia, Bulgaria, analyzes the factors related to the promotion of the Chinese Proficiency Test (HSK) and on this basis presents a few feasible recommendations to the benefit of the Chinese Proficiency Test (HSK).

Keywords: Bulgaria; Confucius Institute Sofia; Chinese Proficiency Test (HSK) current state

Published Date: January 2018

Published Online: $31^{\text {st }}$ January 2018

\section{Introduction}

As China's strength is gradually growing and the country is becoming increasingly influential globally, the interest towards Chinese among Bulgarians is also rising steadily. In order to adapt to the needs of learners of the language to assess their proficiency level, as well as to meet the requirements of work and studying, in late 2002 the Teaching and Research Section of the Department of Chinese Studies at the University of Sofia started an active communication with the China National Office for Teaching Chinese as a Foreign Language and applied for establishing a Chinese proficiency test center. This application received the approval and positive support of the
China National Office for Teaching Chinese as a Foreign Language, of Associate Professor Alexander Fedotov who was in charge of the foreign relations at the University of Sofia, as well as Professor Ge Zhiqiang from the Education group at the Chinese Embassy in Bulgaria. In September 2003 the University of Sofia started organizing the Chinese Proficiency Test and established a "Bulgarian Chinese Proficiency Test Center". This was the earliest such test center ever opened on the territory of the Balkan Peninsula. The first test event included tests on Chinese proficiency in two grades, basic and elementary-intermediate, and was attended by 19 persons (among whom were 3 Macedonian students). Having received meticulous guidance from the lecturers from the University of Sofia, the attendees earned outstanding results and all received Chinese Proficiency Test certificates. The success of this first test event not only stimulated the interest of the students of Chinese Studies at the University of Sofia towards learning Chinese but also provided a major boost to neighboring nations in terms of Chinese language teaching.

\section{A retrospection and analysis on the Chinese proficiency test (HSK) at the Confucius Institute in Sofia}

The Confucius Institute in Sofia was founded in 2007 and took over the work related to HSK test organization. On April 17 $17^{\text {th }}, 2010$ the Confucius Institute in Sofia organized the first ever "New HSK Test" at the Center for Eastern Languages and Cultures of the University of Sofia, with the total 
number of attendees at 78, covering all levels of the test from $1^{\text {st }}$ to $6^{\text {th }}$. This was the year with the highest number of attendees to date. Participants who took the test included university and middle school students from all parts of the country as well as people learning Chinese on their own as a hobby. In order to help the attendees adapt to the new test format and requirements and earn high results, the University of Sofia ran some preparation classes before the examination. The tutors encountered problems due to the fact that they were preparing their students for the new Chinese test with no new teaching materials and made strenuous efforts to compile their own materials after researching the test syllabus provided for each level.

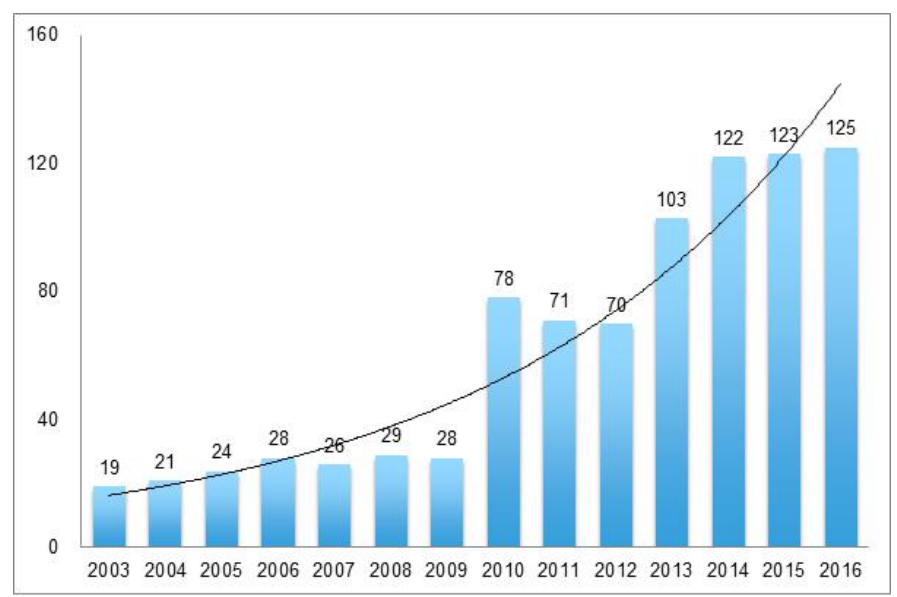

Figure 1 Number of Attendees of the Chinese Proficiency Test (HSK) at the Confucius Institute in Sofia 2003-2016, No of persons

The author of this paper has been working as a tutor in Chinese language at the Confucius Institute in Sofia from January 2013 until present day and for the entire period mentioned has been responsible for test-related work such as registration and proctoring, and has organized and analyzed the Confucius Institute in Sofia HSK archives over the years and derived statistical data therefrom.

Between 2003 and 2009, the University of Sofia and the Confucius Institute organized a total of 7 HSK exams, attended by 196 persons. As can be seen from Fig.1, between 2003 and 2009, the number of attendees remains relatively stable from year to year. This shows that before 2003 there were already a sizeable number of students of Chinese in Bulgaria who had acquired a certain level and they needed to attend the test for language proficiency, so the establishing of the HSK Test Center was most necessary. After 2010, there were two notable sharp rises in the number of HSK attendees, from 2010 to 2012 when it reached 220 and from 2013 to 2016 when it was 345 the rate of growth accelerated rather rapidly. As of October 2016, the total accumulated number of attendees of the HSK exam has reached 761 .

From the number of persons attending the Chinese Proficiency Test (HSK) seen on Fig.1 and the development of the proportion between the number of students at the Confucius Institute in Sofia shown on Fig. 2, it is visible that the future development and spread of the HSK test in Bulgaria still has a huge potential. Currently the proportion stands at around $12: 1$, that is, for each 12 persons studying Chinese there is only one who is attending the HSK, with the numbers likely displaying a positive trend. How to further popularize the Chinese language in Bulgaria with the help of the new HSK amid the already rising interest among Bulgarian students is a question which the Confucius Institute in Sofia should consider carefully and take practical actions towards. The new HSK introduced in 2010 no longer assigned a certain level according to the test result earned by each participant; instead for levels HSK1, 2, 3 and 4 a passing minimum was introduced (180 pts for passing the exam, out of a maximum of 300 pts), and for the two other levels only the number of points started to be reported (with the passing line at 180 pts for levels 5 and 6). Because of this, from 2013 until 2016 when presenting the statistics for the results of the new HSK test we shall only account for the number of persons who passed the test successfully based on the passing minimum of 180 pts. Table 1 below shows that for the three years in question the share of passing attendees for levels 1 and 2 was very high, reaching $100 \%$ at times, then for level 3 it reached $97 \%$ and for level $4-67 \%$ (levels 5 and 6 are not accounted for here). A study on the test papers of 68 attendees in 2015 reveals the following: the attendees at HSK level 1 were 15, all of whom took the exam for the first time; 20 persons took part in HSK 2, 5 of whom attended an 
HSK test for the first time, while for the remaining 15 it was at least the second experience with the exam. For all of those 33 persons who attended HSK level 3 it was at least the second time when they sat for an HSK test. Level 1 of the new HSK test mostly attracted beginner students who only got in touch with Chinese tests for the first time, they visited preparation classes and made special efforts for the purpose. Meanwhile as Table 1 shows between 2013 and 2015 the share of passing attendees at level 4 has remained relatively low. As the level of the new HSK goes higher, the division between test participants becomes deeper.

\begin{tabular}{|c|c|c|c|c|c|}
\hline $\begin{array}{l}\text { Test } \\
\text { Level }\end{array}$ & & $\begin{array}{c}\text { Level } \\
1\end{array}$ & $\begin{array}{c}\text { Level } \\
2\end{array}$ & $\begin{array}{l}\text { Level } \\
3\end{array}$ & $\begin{array}{c}\text { Level } \\
4\end{array}$ \\
\hline \multirow{3}{*}{2013} & No of Attendees & 9 & 14 & 30 & 25 \\
\hline & $\begin{array}{c}\text { No of Attendees } \\
\text { Passed }\end{array}$ & 8 & 13 & 26 & 16 \\
\hline & $\begin{array}{c}\text { Share of } \\
\text { Attendees Passed }\end{array}$ & $89 \%$ & $93 \%$ & $87 \%$ & $64 \%$ \\
\hline \multirow{3}{*}{2014} & No of Attendees & 21 & 15 & 29 & 24 \\
\hline & $\begin{array}{c}\text { No of Attendees } \\
\text { Passed }\end{array}$ & 21 & 14 & 28 & 14 \\
\hline & $\begin{array}{c}\text { Share of } \\
\text { Attendees Passed }\end{array}$ & $100 \%$ & $93 \%$ & $96 \%$ & $58 \%$ \\
\hline \multirow{3}{*}{2015} & No of Attendees & 15 & 20 & 33 & 12 \\
\hline & $\begin{array}{c}\text { No of Attendees } \\
\text { Passed }\end{array}$ & 15 & 20 & 32 & 8 \\
\hline & $\begin{array}{c}\text { Share of } \\
\text { Attendees Passed }\end{array}$ & $100 \%$ & $100 \%$ & $97 \%$ & $67 \%$ \\
\hline
\end{tabular}

Table 1 Statistical Data on the Number of Total Attendees and Attendees Passed at HSK Levels 1-4, 2013-2016

The following factors have influenced the Chinese Proficiency Test (HSK) data presented above in Fig.1 and Table 1:

1.1 The influence of the development of the Confucius Institute in Sofia on the Chinese Proficiency Test (HSK)

The Confucius Institute in Sofia was founded in June 2006, and was the first Confucius Institute established in the Balkans region. Ever since its opening it has been offering a variety of Chinese studying classes for each level, including beginner and intermediate groups, improvement classes, classes following the Great Wall study course etc. With a lot of strenuous efforts and having undergone a long development, the Confucius Institute in Sofia has now established classes covering all age ranges from children to adults, attracting a large number of students interested in the language and culture of China. The number of people signing up for such courses has become significant and has continued growing annually, while the Institute has also been actively developing its subordinate learning locations and Confucius classrooms. As of September 2016, the number of Chinese development learning locations has reached 11 , the number of Confucius Classrooms has grown to five, while the people teaching Chinese have reached 43 and the aggregate number of enrollments has gone up to 1500 . The rising number of the students of Chinese has also strongly boosted the number of those attending the HSK examination. Through taking the exam, these learners can not only test the level that their language skills have reached but can also improve their competitiveness when applying for work or studies. Besides, while there is a permanent inflow of new attendees at the basic levels, some of the students of Chinese have accumulated a broader understanding of the language over time and with the help of their acquired experience from their previous attendance of the HSK have moved forward towards higher levels of Chinese language proficiency.

\section{0}

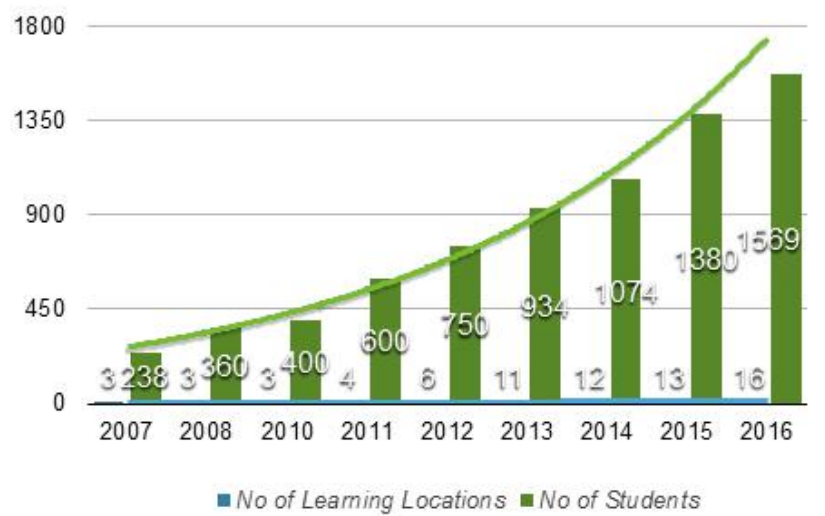

Figure 2 Number of Confucius Institute in Sofia Learning Locations and Learners, 2007-2016 


\subsection{The influence of introduction of the new HSK}

The new HSK is divided into 6 levels, which has turned out to be more attractive for the Bulgarian learners of Chinese. Each of the levels has a clearly defined goal and examination content, and furthermore corresponds to the requirements of the respective levels defined by the "Common European Framework of Reference for Languages" (CEF) (see Table 2). This way not only is the division in levels according to difficulty defined more clearly and the examination targets set in a more direct manner, but also for the Bulgarian attendees who are still studying at schools or universities it is very easy to convert the result earned by them at the HSK test into credits earned from foreign language classes on the base of the standards set by the CEF, which can not only help them achieve their study goals but also improve their competitiveness in their pursuit in advancement with their studies. The old HSK was divided into three levels: beginner, elementaryintermediate and advanced, with each of divided into three different sublevels of $A, B$ or $C$ based on the test scores. This approach has a clear disadvantage since when the result is formulated as "Elementary-Intermediate Level B", it is not sufficiently easy to comprehend and the students can easily get confused when making their choice for taking a higher level of the test. Some students have stated in interviews that after acquiring the certificate for Beginner level B, they were confused whether they should attend again the beginner level test to earn Beginner level A or to rush straight onto the higher Elementary-Intermediate level test. This has resulted in a slowdown in the growth of the number of attendees of the ElementaryIntermediate exam.

\begin{tabular}{|c|c|c|c|c|c|c|c|}
\hline New HSK & $\begin{array}{l}\text { Vocabulary } \\
\text { (number of } \\
\text { words) }\end{array}$ & $\begin{array}{l}\text { International } \\
\text { Chinese } \\
\text { Proficiency } \\
\text { Standard }\end{array}$ & $\mathrm{CEF}$ & Examination Content & $\begin{array}{l}\text { Number of } \\
\text { Questions }\end{array}$ & $\begin{array}{l}\text { Exam } \\
\text { Time }\end{array}$ & $\begin{array}{l}\text { Total } \\
\text { Score }\end{array}$ \\
\hline HSK Lvl 6 & $5000+$ & Lvl6 & $\mathrm{C} 2$ & Listening, reading, writing & $50+50+1=101$ & 135 & 300 \\
\hline HSK Lvl 5 & 2500 & Lv15 & $\mathrm{C} 1$ & Listening, reading, writing & $45+45+10=100$ & 120 & 300 \\
\hline HSK Lvl 3 & 600 & Lvl 3 & B1 & Listening, reading, writing & $40+30+10=80$ & 85 & 300 \\
\hline HSK Lvl 2 & 300 & Lvl 2 & A1 & Listening, reading & $35+25=60$ & 50 & 200 \\
\hline HSK Lvl 1 & 150 & Lvl 1 & $\mathrm{~A} 2$ & Listening, reading & $20+20=40$ & 35 & 200 \\
\hline
\end{tabular}

Table 2 New HSK Exam Structure for Each Level and Correspondence between the "International Chinese Proficiency Standard" and the Common European Framework of Reference for Languages (CEF)

\subsection{The popularization of HSK}

As the relations between China and Bulgaria in aspects such as politics, economics, culture and diplomacy are deepening and the contacts between the two nations are becoming closer, the popularity of Chinese is spreading rapidly in Bulgaria. On one hand, many local companies and cultural organizations are hoping to boost their development by expanding their activity towards China, and as a result Chinese is becoming a necessary communication tool for the purpose; on the other, many Chinese migrants and tourists visit the Bulgaria and now occupy a certain position in the local society, and, in order to make those one's customers, one also needs to be able to use Chinese. All of these reasons have made it a necessity for local people to acquire some proficiency in Chinese. And HSK, being an official international standardized language test organized by China and already doubtlessly enjoying some level of reputation, is seen by Bulgarians as an important standard for testing their Chinese language skills. Furthermore, Bulgarian students can 
only apply for scholarships and advanced academic education at Chinese universities and colleges by means of the HSK exam. The employment situation in Bulgaria is rather tight and earning a certificate for Chinese proficiency can give one a certain advantage over the other candidates, and besides, in the long run, Bulgaria is going to need a large number of local skilled professionals having the ability to use Chinese.

\section{A poll survey and analysis on the attitude towards the Chinese proficiency test (HSK)}

In order to get a better idea of the attitude and views of the HSK test attendees at the Confucius Institute in Sofia, Bulgaria, the author of this paper conducted a poll survey on the topic. A total of 60 questionnaires were distributed among 15 students learning beginner Chinese at the Confucius Institute in Sofia, 15 students from the Chinese Studies program at the University of Sofia and 30 high school students from the $18^{\text {th }}$ High School in Sofia, the High School of Mathematics in Varna and a high school in Plovdiv, and in the end 55 of those were returned. Among the polled students 30 had been studying Chinese for more than one year and a half, the longest period being 3 years. 14 had been studying for less than one year, with the shortest period of studying being 3 months. The poll included 14 questions and the results' analysis will be hereby presented.

The following table summarizes the data regarding the familiarity of the learners with the Chinese Proficiency Test and their attendance history.

\begin{tabular}{|c|c|}
\hline Poll Question & Results \\
\hline Do you know about the HSK? & 45 persons knew $(81.8 \%), 10$ persons did not know(18.2\%) \\
\hline How did you discover about the HSK? & $\begin{array}{l}31 \text { persons were told by their teachers or friends( } 68.9 \%), 14 \text { persons } \\
\text { discovered it online }(25.4 \%)\end{array}$ \\
\hline What do you think the HSK is? & $\begin{array}{l}25 \text { persons thought it is a language proficiency test }(45.5 \%), 20 \\
\text { persons thought it is a certificate for application for advanced studies } \\
\text { in China ( } 36.3 \%), 10 \text { persons had no idea ( } 18 \%)\end{array}$ \\
\hline $\begin{array}{l}\text { How many times have you attended } \\
\text { HSK? }\end{array}$ & $\begin{array}{l}21 \text { persons had attended once (38\%), } 17 \text { persons had attended twice } \\
\text { or more }(30.9 \%), 17 \text { persons had never attended (30.9\%) }\end{array}$ \\
\hline $\begin{array}{l}\text { What level(s) of HSK have you } \\
\text { attended? }\end{array}$ & $\begin{array}{l}15 \text { persons had attended HSK IVI } 1 \text { ( } 27 \%), 15 \text { persons HSK IVI } 2 \text { ( } 27 \%) \text {, } \\
5 \text { persons HSK IVI } 3(9 \%), 4 \text { persons HSK IVI } 4 \text { (7\%), } 3 \text { persons HKS IVI } 5 \\
(5 \%), 1 \text { person HSK IVl } 6(1.8 \%), 12 \text { persons had never attended HSK } \\
(12.7 \%)\end{array}$ \\
\hline Do you think the HSK is useful? & $\begin{array}{l}30 \text { persons believed it can improve their confidence in learning } \\
(54.5 \%), 15 \text { persons believed it gave them a chance to go to China } \\
(27.3 \%), 3 \text { persons thought it was not useful }(5.4 \%), 7 \text { persons had no } \\
\text { answer }(12.7 \%)\end{array}$ \\
\hline
\end{tabular}

The poll results show that for the majority of the students after studying Chinese for 3 months $81.8 \%$ of them are aware of the HSK exam and most of them have heard about it from their teachers. Some of the HSK attendees among them did it to test their skills, to improve their confidence, and there were also some who did it because they wanted to receive a scholarship for studying at a Chinese university. The Embassy of China in Bulgaria offers annually to Bulgarian learners of Chinese some government scholarships for China, while the headquarters of the Confucius Institute has since 2006 offered its own scholarships for learners of Chinese at the Confucius Institute of Sofia. Besides, the annual competition 
"Chinese Bridge" also provides a number of scholarships for studying in China. The attendance of all of these three events and the selection of candidates all require some level of Chinese proficiency and a test result from HSK, which has further supported the growth in numbers of HSK attendees.

The following table shows the poll results regarding the exam preparation offered by the Confucius Institute in Sofia and the students' views on the examination questions.

\section{\begin{tabular}{|l|l}
\hline Poll Question & Results \\
\hline
\end{tabular}}

How do you get prepared for HSK?

15 persons made a revision by themselves (27.2\%), 17 persons attended preparation classes with a teacher (30.9\%), 13 persons exercised with their friends (23.6\%), 9 persons gave no clear answer $(16.3 \%)$

What kind of preparation class did you attend before HSK?

16 persons attended some classroom activities (29\%), 28 persons attended classes at the Confucius Institute (50.9\%), 11 persons did not attend preparatory classes

How much time did the preparatory classes occupy?

10 persons less than a month (38.4\%), 16 persons one month or more (61.5\%)

Do you think the training and materials provided by the teaching organization were sufficient?

12 persons thought they were sufficient (21.8\%), 19 persons were neutral (34.5\%), 10 persons thought they were not sufficient (18.2\%), 14 persons gave no specific answer (25.4\%).

Where did you acquire learning materials from?

24 persons got them from the Confucius Institute (43.6\%), 4 persons purchased materials from China (8\%), 6 persons acquired them online (10.9\%), 5 persons used no preparatory materials (9\%), 16 persons thought HSK materials ware hard to acquire.

Do you think the exam preparation was useful? Does any aspect need improvement?

32 persons said it was very useful and very necessary (58.2\%), 20 persons said it was useful and necessary $(36.4 \%), 3$ persons had no opinion (5.4\%)

How do you find the test questions in HSK?

11 persons thought they were very easy (20\%), 18 persons thought they were of normal difficulty (32.7\%), 16 persons thought they were too difficult (29\%), 10 persons had no opinion (18.2\%)

What part of the HSK do you find to be the hardest?

19 persons thought it was listening (34.5\%), 13 persons thought it was writing (23.6\%), 12 persons thought it was reading (21.8\%), 12 persons had no answer.

The survey results show that the preparation work carried out by the Confucius Institute is viewed positively by the learners, but there are also some problems such as the short time and the lack of revision materials, and therefore in the future the time for preparation could be extended and new materials should be provided in accordance with the specific conditions. Students generally think that the HSK exam is not too difficult. Students who have studied Chinese less than one year believe that listening is the most simple testing aspect, writing is the hardest one, and the reading part is somewhere in between; while those who have studied for more than year think that writing is the easiest, listening is 
the hardest, and reading is the second hardest part of the test. Since students at beginner level are just getting in touch with Chinese and are not familiar with the writing system, it seems to them that the Chinese characters are the most difficult aspect. Meanwhile those who have an experience of studying Chinese for more than a year have already grasped some basic knowledge on characters, but because of the absence of language environment for studying and exercising they have more difficulties with listening and speaking. The author of the current paper interviewed some attendees immediately after the HSK test which took place on October $16^{\text {th }}, 2016$ in order to inquire into their recommendations regarding the exam. They hope that guidance on the HSK test could penetrate into daily teaching activities, that adequate materials can be provided for the preparation, and that the time for preparatory classes before the test can be extended; they also suggest that more resources be used for the promotion of the HSK and that the test provides more opportunities for the test attendees to visit China for studying or attending language specialization.

\section{Recommendations on further improving the Chinese proficiency test (HSK) at the Confucius Institute in Sofia}

Regarding the current situation, it could be said that the HSK in Bulgaria is following a favorable trend of development; however it should also be noted that while analyzing the situation around HSK and conducting the survey on the popularization of the test, some problems have been discovered. Those problems tend to inevitably occur in the development of this type of standardized international exams. In Bulgaria, the HSK has essentially grown out of nothing and has quickly made the transition from its initial phase into the stage of development. Some questions which should be seriously considered by the Confucius Institute in Sofia include how to discover the problems from the past experience and how to summarize and draw conclusions from it in order to bring about gradual improvement. The author of the paper will hereby present a few feasible suggestions on the popularization of the HSK based on the data and information gathered through the survey and interviews, while also taking into consideration the specific local conditions. The Confucius Institute in Sofia should act in the following four directions:

\subsection{Boosting promotion work and diversifying promotion channels while attaching great importance to collecting data and information}

Among learners of Chinese as a second language, HSK plays an important role: it has an impact not only on the professional approach of teachers of Chinese to foreigners but also on the studying behavior of the broad mass of learners. Therefore, the Confucius Institute of Sofia should attach some special importance to collecting HSK data and information from the different learning centers which should be systematically analyzed and assessed. The impact of the exam on Chinese teaching in Bulgaria, on teaching personnel, attendees and the society on the whole should be evaluated and on the basis of this the level of teaching of Chinese at all teaching learning centers in Bulgaria should be boosted in a targeted manner, the scope of the HSK should be expanded and improve its quality improved. Although the number of HSK attendees in Bulgaria has been constantly rising over the last years, proportionally to the number of total students of Chinese in the country it still represents a rather small share. The current promotion channels are chiefly the Chinese learning locations, and the target crowd is only the students at these locations. Besides those are restricted by the availability of audio devices and other hardware at the learning locations and this together with the lack of variety of the promotion channels is negatively impacting the effectivity of promotion. In the future, the promotion of HSK should also include some media channels such as the internet, press, TV or TV advertisement; except for the teachers at the learning centers presenting the exam and recommending to students to take part in it, HSK attendees whom the exam helped earn Chinese government scholarships, Confucius Institute 
Scholarships or Chinese Bridge student scholarships should also be sharing their experience from the test, thus also strongly boosting its promotion.

\subsection{Providing more materials for HSK preparation to Bulgarian attendees and writing new such materials suitable for the local exam takers.}

Currently, the specialized materials which Bulgarian learners can acquire for HSK preparation are the editions of the "Chinese Proficiency Test Syllabus", compiled by the Hanban Institute and published by The Commercial Press, and those can only be found through the Confucius Institute. In our survey we asked the learners about the ways and problems for finding HSK preparation materials, and 24 persons (43.6\%) said they found such materials through the Confucius Institute, 4 persons bought them from China (8\%), 6 persons ordered them online (10.9\%), and 5 persons did not get any specialized HSK materials. Out of the 55 people who took part in the poll, 16 (or $29 \%$ ) believe that HSK-related materials are hard or very hard to acquire. The students who were interviewed also stated that such materials are very difficult to find In Bulgaria, and even older materials from some years ago are very rare, so if the Confucius Institute had not provided the exam syllabus, they would have had no idea how to proceed with studying. In this aspect, the Institute largely facilitated learners, but it is still very far from meeting all of their needs. The Confucius Institute in Sofia should support learning centers and Confucius classrooms by purchasing HSK guidebooks, workbooks, dictionaries etc. and organize Chinese teachers and local linguists to form teams for composing HSK questions so that they can be able to publish Bulgarian versions of HSK guidebooks. Cooperation should be sought with local publishing and print organizations for establishing some centers for HSK material publishing so new materials can be provided for the attendees at the test center. Hanban could also consider publishing more HSK materials in other languages in the future in order to meet the needs of overseas learners of Chinese in all countries and regions around the world. It can be expected that as HSK is spreading out throughout the world these needs will result in the formation of a broad market. The work done on Chinese teaching in Bulgaria has already established a good base for the future development of HSK, and at the same time the opposite is also true - the HSK has expanded the scope of Chinese teaching in Bulgaria. The authoritativeness of the HSK and the practical benefits of the HSK certificates have inspired many Bulgarian people who are interested in studying Chinese to go even deeper in their studies of the language.

\subsection{Introducing an exam for primary and middle schools (Youth Chinese Test, YCT)}

The learning locations overseen by the Confucius Institute of Sofia currently include 10 primary and middle schools and 3 Confucius classrooms, with a total of over 700 students studying Chinese in them, about half of whom are taking lessons in Chinese by visiting interest-oriented classes. The turnover of these students in interest-oriented classes is rather high. Dealing with the loss of students of Chinese and learning locations should be attached some special importance to. The YCT exam should come to the focus of the Institute, which should actively promote the Chinese Proficiency Test for Primary and Middle Schools, known as Youth Chinese Test (YCT) at all learning locations. Such promotion of the YCT could boost the self-confidence of students and their sense of prestige, help them retain their interest towards the language, and their results can be used by them as a reference ground for assessing their Chinese skills and further improve them in the future. The YCT results could also serve as a reference for the classes in Chinese they are taking at school, and provide to schools and organizations which teach Chinese a reference basis for the effectiveness of their teaching work. Furthermore the results can be used as a reference for attending HSK tests. The rising number of YCT attendees will directly result in an increase in the number of people taking the HSK in Bulgaria as well. The new HSK is divided into 3 types and six levels, with the so-called three types include the new HSK test, the new Youth Chinese Test (YCT) and the Business Chinese Test 
(BCT). The levels are 6 for the new HSK, 4 for the YCT and 5 for the BCT. The new Youth Chinese Test (YCT) is a standardized international Chinese proficiency test, assessing the Chinese skills used in everyday life and in studying of primary and middle school students whose first language is not Chinese. It follows the principle of combining teaching, learning and testing, and is designed in a way to closely match the development of Chinese teaching in primary and middle schools around the world as well as the teaching materials used. It has been successful at stimulating learning and teaching through testing. Its introduction into primary and middle schools in Bulgaria would be largely beneficial.

\subsection{Establishing specialized HSK preparatory groups, stabilizing teaching staff and preparing local teachers}

Taking a look at the qualities of Bulgarian teachers of Chinese reveals that most of them lack in specialized knowledge in regard of the HSK, have no specialized preparation in terms of HSK testing, and there is an overall lack of high-quality, well-trained instructors with developed HSK preparation skills. Most of the teachers doing HSK-related work are concentrated in the Confucius Institute, while in the schools and learning locations there is a lack of staff to support the Institute in terms of HSK organization. These problems inevitably have a negative impact on the results of Bulgarian HSK attendees. According to the Hanban Institute's regulations, teachers of Chinese outside the country serve for a term of 2-4 years, while volunteer instructors serve for a term of 1-3 years. Currently the number of Chinese teachers sent by the state to the Confucius Institute of Sofia is 8 and the volunteer instructors are 13 . The main 'force' of Chinese teaching are the volunteers, but most of them are doing graduate studies and due to their need to complete these studies and their life and career goals the vast majority of them spend in Bulgaria less than 2 years. The Confucius Institute in Sofia already has more than 10 local teachers of Chinese but comparing to the growth in number of students at the Institute, local teachers are falling far behind and cannot meet the needs of the Institute for local teaching staff. Facing these circumstances, the Confucius Institute should proceed by taking the practical situation into consideration when solving the problems: first, there are already a few universities in the country that have established bachelor and master programs in Chinese, and the students who attend them are not only fluent in the local language but also have a background of systematic studying of Chinese, so they could get fully engaged in Chinese teaching. The Institute should start a specialized pre-employment funded training program for protecting the local teaching staff, thus stabilizing its team of Chinese instructors and preparing its local teachers, this way making sure that all learning locations have adequately prepared teaching professionals and eventually further boosting the popularity of Chinese learning and the HSK test in Bulgaria. Secondly, tutors of Chinese should improve their teaching skills and the skills related to the high-level HSK preparation, expanding the specialized knowledge that an excellent teacher of Chinese is required to possess. The Confucius Institute should establish a mechanism involving a group especially responsible for the HSK and prepare teachers who specialize in HSK-related work. The HSK instructors should conduct specialized research on the information and knowledge concerning the exam and communicate effectively with the teachers from each learning location responsible for HSK and actively seek the support and help of those instructors and the local teachers. The teachers of Chinese should perform some preparation activities for the local teachers assisting for the exam, present the test procedures and regulations, and such training should be carried out on a regular basis with each Chinese teacher and volunteer passing the relay forward onto the next level.

The Chinese Proficiency Test (HSK) is an essential part of the popularization of Chinese studying and is becoming increasingly important for the Confucius Institute. The main goal of the survey on the Confucius Institute in Sofia HSK is to provide a basis for the further popularization and development of the test in Bulgaria. By researching and analyzing the current state and popularization of the Confucius 
Institute in Sofia HSK test we discovered that the main factors restricting its development is the availability of qualified teachers, teaching materials, examination resources and basic equipment. The sustainable "both fine and fast" positive development of HSK in Bulgaria needs more variable and more intense promotion channels; stabilizing the team of qualified instructors and raising local teaching talent; promoting HSK in Bulgaria by teaching according to the students' abilities and undertaking actions according to the local conditions. The author of the paper hopes that the recommendations proposed on the grounds of the analysis of the current state of the HSK in Bulgaria and the research on the factors influencing its promotion could serve for a basis for some future strategies for the promotion of the HSK in Bulgaria, for Chinese learning in the country and the development of the Confucius Institute in Sofia.

\section{References}

[1] Hanban/ Confucius Institute Headquarters. New HSK Syllabus [M]. Beijing, The Commercial press, 2009.

[2] Xu Yumin. The Role of HSK in the Development of Chinese Teaching in Italy [A]. Selected Papers from the $8^{\text {th }}$ International Chinese Teaching Conference [C], Beijing: Chinese High Education Press, 2007.

[3] Zhang Jinjun. Research Report on the New HSK. [J]. Chinese Exam, 2010 (9): 38-43.

[4] Liu Lianli. A Study on Chinese Proficiency Testing [M]. Beijing: Beijing Language and Culture University Press, 1998
[5] Liu Quan, Wu Xiukui. A Survey Analysis on the HSK at the Confucius Institute at the North-South University in Bangladesh [J]. Journal Of Chuxiong Normal University. 2013, 12:42-47

[6] Sun Yunhe. The Promotion and Development of HSK in Italy: Analysis on the Test Situation at the University of Rome over 6 Years. [J]. Science Journal of Hunan Normal University Pedagogics Department, 2011, 02:81-85.

[7] Zhao Xun, Zhang Lingyan. Questions, Policies and Significance of the YCT Exam in Nepal. Journal of Language and Literature Studies, 2013, 13:122-125

[8] Dong Shuhui: "50 Years of Chinese Teaching in Bulgaria", Sofia Jade Press, June 2005

[9] CEF (A Common European Frame-work of Reference for Languages : Learning, Teaching , Assessment), proposed in 2001 by the European Committee and published in 2003. The framework is a foreign language teaching, learning and assessment standard, aimed at providing a common base and reference for teaching languages in Europe and the related course design, syllabus and textbook writing and language tests.

\section{Main Websites}

Beijing Language University HSK Center: www.hsk.org.cn Hanban/Confucius Institute Headquarters: www.hanban.org Hanban HSK Service Network: www.chinesetesting.cn 